\title{
Welche Integrationstheorie braucht Europa?
}

\author{
Jürgen Neyer*
}

\section{Von Maastricht zum Fußnotengipfel}

Der Vertrag von Maastricht wurde Anfang der neunziger Jahre als Durchbruch zu einer neuen Qualität von Integration gefeiert. Die Unionsbürgerschaft und die europäische Hymne galten als Vorboten einer möglichen zukünftigen Nationalstaatswerdung Europas. Die nur wenige Jahre später eingeführte gemeinsame Währung, die Grundrechtecharta und das Projekt des Verfassungsvertrages sollten Europa aus den Fesseln gouvernementaler Selbstblockade befreien und ein demokratisches, effizientes und bürgernahes Europa begründen. Diese hochfliegenden Hoffnungen liegen inzwischen als Trümmer auf dem Boden der europäischen Realität. Der ,Fußnotengipfel ${ }^{{ }^{\circ}}$ vom Juni 2007, der fast so viele Sonderregelungen, Klarstellungen und Ausnahmen wie Übereinkommen produziert hat, zeigte in aller Klarheit, dass die Stimmung in Europa heute eher von Misstrauen und Zögern als von Euphorie geprägt ist.

Die aktuelle Krise Europas ist nur oberflächlich gesehen eine Krise divergierender Regierungspositionen. In ihrem tiefer liegenden Kern bringt sie den gesellschaftlichen Unmut darüber zum Ausdruck, dass das seit vielen Jahren bemühte Versprechen eines bürgernahen Europas nicht eingelöst wurde, sondern dass die politische Praxis Europas nach wie vor in weiten Bereichen eine Mischung aus Technokratie und interexekutiver Verhandlung darstellt. ${ }^{2}$ Europa ist politisch erwachsen geworden und damit auch der Möglichkeit einer technokratisch-bürokratischen Legitimation entwachsen. Die europäischen Öffentlichkeiten sind heute nicht mehr bereit, sich vorbehaltlos der Effizienz technokratischer Expertise anzuvertrauen, sondern bewerten Europa mit den sehr viel anspruchsvolleren Maßstäben der demokratischen Legitimation.

Das gestiegene Selbstbewusstsein der europäischen Öffentlichkeit hat nicht nur den Integrationsprozess, sondern gleichzeitig auch die Integrationstheorie in die Krise gestürzt. Die wichtigsten integrationstheoretischen Ansätze wie der Neofunktionalismus, der Intergouvernementalismus und der Mehrebenenansatz verfügen über kein angemessenes analytisches Vokabular, um die neue Rolle der Öffentlichkeit und die Kluft zwischen Europa und seinen Bürgern erfassen zu können. Die Beschreibung und kritische Analyse der Europäischen Union benötigt daher eine erneuerte Integrationstheorie, die gesellschaftstheoretisch aktualisiert und auf die veränderten Rahmenbedingungen angemessen eingestellt ist.

* Prof. Dr. Jürgen Neyer, Professur für Politikwissenschaft, Europa-Universität Viadrina. Der Autor dankt den beiden anonymen Gutachtern.

1 Vgl. zum Begriff auch Annegret Bendiek: Die GASP nach dem „Fußnotengipfel“, SWP-Aktuell 2007/A 42, Juli 2007, Berlin.

2 Die Daten des aktuellen Eurobarometers weisen darauf hin, dass nur gut die Hälfte aller Befragten die Mitgliedschaft in der Europäischen Union für eine gute Sache hält und den europäischen Institutionen vertraut (Europäische Kommission: Eurobarometer 67, Public Opinion in the European Union, 2007, S. 15-22). Diese skeptische Einstellung ist zwar nicht neu, muss aber vor dem Hintergrund des historischen Erfolges der Einbindung und Stabilisierung Mittel- und Osteuropas heute noch mehr als früher beunruhigen. Die Fragen des Eurobarometers sind zudem sehr allgemein gestellt. Es muss davon ausgegangen werden, dass konkretere Fragen wie etwa die nach dem Verständnis und der Nachvollziehbarkeit europäischer Politikprozesse noch weniger positive Antworten erhalten würden. 


\section{Die Krise der Integrationstheorie}

Neofunktionalistische Theorien sind heute noch immer ein wichtiger Bestandteil der Integrationstheorie. ${ }^{3}$ Bei aller Unterschiedlichkeit der verschiedenen neofunktionalistischen Ansätze im Einzelnen, treffen sich die meisten doch in der Gemeinsamkeit, Integration als einen unpolitischen Prozess zu verstehen, der von der internen Logik funktionaler Angemessenheit getrieben wird. Ernst Haas betonte zwar, dass gesellschaftliche Akteure von wesentlicher Relevanz sind und dass der Integrationsprozess damit - anders als noch bei Mitrany ${ }^{4}$ nicht als technokratisches Projekt missverstanden werden dürfte. Gleichzeitig aber betonte Haas die sogenannten ,Spill-over Effekte', die zu einem quasi-automatischen Prozess der Übertragung immer weiterer Kompetenzen von der nationalstaatlichen auf die supranationale Ebene führen würden. ${ }^{5}$ Die Mitgliedstaaten wären bereit, die hiermit einhergehenden Kompetenzübertragungen zu akzeptieren, da sie letztlich an einer funktional leistungsfähigen Politik interessiert seien.

Die heroisch unpolitischen Annahmen funktionalistischen Denkens sind heute nur noch vor dem Hintergrund der Schrecken des Zweiten Weltkrieges und als grundsätzlich normative Positionen verständlich. Der Nationalstaat und die unkontrollierbare demokratische Öffentlichkeit galten in der Nachkriegszeit als grundlegende Probleme der internationalen Politik, die durch eine kühle technokratische Politik im Zaum gehalten werden müssten. ${ }^{6}$ Der Begriff der Gesellschaft im Sinne eines kritischen und kontrollierenden Gegenübers staatlicher Politik war dem Neofunktionalismus hingegen fremd. Es kann daher heute auch kaum verwundern, wenn Analysten, die in der Tradition des Neofunktionalismus stehen, von der Ablehnung des Verfassungsvertrages überrascht sind, die resultierenden ökonomischen und politischen Ineffizienzen beklagen und entweder eine Wiederholung der Referenden fordern oder - noch besser - ein möglichst weitgehendes Vermeiden jeglicher gesellschaftlicher Konsultation. Das neofunktionalistische Politikverständnis ist von einer grundlegenden Skepsis gegenüber dem konstruktiven Potenzial der Öffentlichkeit geprägt.

Auch das Demokratie- und Öffentlichkeitsverständnis des Intergouvernementalismus mutet heute merkwürdig überholt an. Der liberale Intergouvernementalismus betont zwar ebenso wie der Haas'sche Neofunktionalismus die Rolle einer pluralistischen Gesellschaft. Gleichzeitig aber macht er die Wirkungsmächtigkeit von gesellschaftlichen Akteuren davon abhängig, dass sie in der Lage sind, gouvernementale Präferenzen lobbyistisch zu beeinflussen und verengt das innenpolitische Handeln von Regierungen darauf, vorherrschende Gewerkschafts- oder Unternehmensinteressen abzubilden. ${ }^{7}$ Gesellschaft erscheint in einer korporatistisch verengten Sichtweise, die nur institutionalisierte Interessen kennt. Diffuse Interessenlagen, nichtorganisierte gesellschaftliche Meinungen und die Öffentlichkeit werden weitestgehend ausgeblendet. Während die klassische Demokratieanalyse uns erwarten ließe, dass jede Regierung schon aus Gründen des Machterhaltes immer versuchen muss, breite Gesellschaftskreise zu erreichen und, volksparteiliche' Politik zu gestalten, ist die Regierung im liberalen Intergouvernementalismus offensichtlich ausschließlich offen für die Wünsche gut organisierter Interessengruppen. Die demokratische Öffentlichkeit und die de-

3 Für eine aktuelle Anwendung auf die Internationalen Beziehungen vgl. Michael Zürn/Martin Binder/Matthias Ecker-Ehrhardt/Katrin Radtke: Politische Ordnungsbildung wider Willen, in: Zeitschrift für Internationale Beziehungen 1/2007, S. 129-164.

4 David Mitrany: A Working Peace System, Chicago 1943.

5 Ernst B. Haas: Beyond the nation-state. Functionalism and international organization, Stanford 1964.

6 Mitrany: Working Peace System, 1943.

7 Andrew Moravcsik: The choice for Europe: Social purposes and state power from Messina to Maastricht, Ithaca 1998. 
mokratieimmanente Notwendigkeit, Politiken öffentlich zu rechtfertigen, tauchen als analytische Kategorie nicht auf. Von einer Integrationstheorie, die in derart gravierender Weise die gesellschaftlichen Grundlagen der Europäischen Union ausblendet, kann nicht erwartet werden, dass sie gesellschaftlich induzierte Krisen vorhersieht und die heutige politische Realität Europas versteht.

Die reduktionistischen Demokratie- und Öffentlichkeitsverständnisse des Neofunktionalismus und des Intergouvernementalismus dürften mit dafür verantwortlich sein, dass die neuere Integrationsforschung sich auf die Suche nach alternativen theoretischen Orientierungspunkten begeben hat. Insbesondere die Konzepte des Mehrebenensystems ${ }^{8}$ und der Europäisierung ${ }^{9}$ haben die Forschung der letzten zehn Jahre stark beeinflusst. Als Mehrebenensystem wird eine politische Konfiguration verstanden, in der die innerstaatliche, die zwischenstaatliche und die supranationale Politik sich wechselseitig ergänzen und ein übergreifendes politisches System bilden. Nationale Regierungen werden zwar als zentrale Akteure behandelt, müssen sich den politischen Prozess aber mit anderen Akteuren teilen. Regieren in Europa wird als eine mehrstufige, funktional differenzierte, nicht hierarchische, durch netzwerkartige Interaktionsstrukturen geprägte Struktur der Steuerung und Koordinierung von Handlungen und Entscheidungen verstanden. ${ }^{10}$ Der Begriff des Mehrebenensystems hat seine wesentliche Stärke darin, den Betrachter für die Komplexität der Europäischen Union zu sensibilisieren. Das Defizit des Konzeptes liegt allerdings darin, dass sich kaum klare Aussagen über politische Prozesse und Politikergebnisse ableiten lassen. Der Begriff des Mehrebenensystems ist nicht viel mehr als eine Metapher, die zwar eine Richtung für die Analyse weist und zu hilfreichen deskriptiven Annäherungen einlädt. Der Begriff ist aber weder gesellschaftstheoretisch angebunden noch in der Lage, empirisch überprüfbare Hypothesen zu generieren.

Ganz ähnlich beinhaltet auch das Konzept der Europäisierung wenig gesellschaftstheoretisch fundierte Inhalte. Es ist ebenso wie das Konzept des Mehrebenensystems eher eine analytische Ausrichtung für die Eingrenzung einer Fragestellung als eine eigenständige Theorie. Die Europäisierungsforschung fragt danach, unter welchen Bedingungen die europäischen Mitgliedstaaten in der Lage sind, die Europäische Union für ihre Politiken zu instrumentalisieren (,uploading of policies`) und wann sie zu welchem Ausmaß europäische Politiken innerstaatlich umsetzen (,downloading of policies $\left.{ }^{6}\right) .{ }^{11}$ Diese Fragen sind für eine verwaltungswissenschaftlich orientierte Europaforschung außerordentlich interessant und haben eine Vielzahl interessanter empirischer Studien hervorgebracht. Beide Fragen sind damit willkommene Bereicherungen für die Integrationsforschung. Gleichzeitig aber können sie das Defizit einer fehlenden Berücksichtigung von Demokratie und Öffentlichkeit nicht beheben. Ganz im Gegenteil. Die zunehmende Verwendung von Konzepten, die den demokratischen Charakter der Mitgliedstaaten der Europäischen Union konzeptionell übersehen, droht die Integrationsforschung von einer Politikwissenschaft abzukoppeln, die sich nicht zuletzt als Demokratiewissenschaft versteht. ${ }^{12}$ Als verwaltungswissenschaftlich verkürzte

8 Markus Jachtenfuchs/Beate Kohler-Koch: Europäische Integration, Opladen 2003.

9 Johan P. Olson: The Many Faces of Europeanization, in: Journal of Common Market Studies 5/2002, S. 921952.

10 Arthur Benz: Politische Steuerung in lose gekoppelten Mehrebenensystemen, in: Raymund Wehrle/Uwe Schimank (Hrsg.): Gesellschaftliche Komplexität und kollektive Handlungsfähigkeit, Frankfurt a. M. 2000, S. 99-126.

11 Tanja Börzel: Pace-setting, foot-dragging, and fence-sitting: Member state responses to Europeanization, in: Journal of Common Market Studies 2/2002, S. 193-214.

12 Ein Verständnis von Integrationsforschung als Demokratiewissenschaft findet sich etwa bei Heidrun Abromeit: Wozu braucht man Demokratie? Die postnationale Herausforderung der Demokratietheorie, Opladen 2002. 
Teildisziplin wird die Integrationsforschung aber kaum den intellektuellen Reiz behalten können, der sie in den letzten Jahren für die Internationalen Beziehungen, die Regierungslehre und die politische Theorie attraktiv gemacht hat.

\section{Deliberative Integrationstheorie}

Die deliberative Integrationstheorie ist ein relativ junger Strang der Integrationsforschung, der sich als Antwort auf ihre aktuellen Defizite versteht. Ihre ursprüngliche Inspiration hat sie aus der empirischen Einsicht bezogen, dass die europäische Politik zu einem erstaunlich hohen Ausmaß von argumentativen und rechtfertigungsbasierten Prozessen geprägt ist. Eine Vielzahl empirischer Studien hat auf argumentative und an der Konsenssuche orientierte politische Prozesse hingewiesen, während Mehrheitsverfahren offensichtlich nur sehr zögerlich verwandt werden. ${ }^{13}$ Beobachter der Europäische Union beschreiben einen ,instinctive recourse to behave consensually" und den allgegenwärtigen Versuch, so viele Staaten wie möglich für eine Einigung zu gewinnen. ${ }^{14}$ Dieser Befund gilt für den Rat genauso wie für andere legislativ tätige Institutionen der Europäischen Gemeinschaft wie etwa den Ausschuss der Ständigen Vertreter ${ }^{15}$ und die meisten Kommissionsausschüsse. ${ }^{16}$ Politische Interaktion ist in der Europäischen Gemeinschaft offensichtlich stark vom Versuch der Verständigung geprägt.

Deliberative Ansätze, die sich mit diesem Phänomen beschäftigen, finden sich in einer ganzen Reihe von Ausprägungen. Das Spektrum reicht von theoretisch-abstrakten Beiträgen, die sich mit der Konzeptualisierung einzelner Begriffe befassen, ${ }^{17}$ bis hin zu empirischen Beiträgen, die versuchen, die Praxis politischer Verständigungsprozesse nachzuzeichnen. ${ }^{18}$ Es finden sich Beiträge, die intergouvernementalem ${ }^{19}$ oder auch supranationalem ${ }^{20}$ Denken verhaftet sind. Schon aus Platzgründen kann hier nicht der Anspruch erhoben werden, einen Gesamtüberblick über die Literatur zu geben. ${ }^{21}$ Im Folgenden soll vielmehr eine Möglichkeit vorgestellt werden, wie deliberative Theorieelemente für die Erklärung wesentlicher Prozesse europäischen Regierens nutzbar gemacht werden können. Die grundsätzliche Idee der hier skizzierten Theorie basiert auf der Annahme, dass politische Prozesse das Produkt nationaler Akteure sind, die ihr Handeln auf die Anreize einer institutionellen Struktur einstellen. Die folgende Darstellung orientiert sich entsprechend an der Dreiteilung von Struktur, Akteur und Prozess.

13 John Peterson: Decision-making in the European Union: Towards a framework for analysis, in: Journal of European Public Policy 2/1995, S. 69-93, hier S. 73: ,Very few important policy-setting decisions are taken without the agreement of all twelve governments to a Commission proposal".

14 Fiona Hayes-Renshaw/Helen Wallace: Executive power in the European Union: the functions and limits of the Council of Ministers, in: Journal of European Public Policy 4/1995, S. 559-582, hier S. 565.

15 Jeffrey Lewis: Is the "Hard Bargaining" Image of the Council Misleading? The Committee of Permanent Representatives and the Local Elections Directive, in: Journal of Common Market Studies 4/1998, S. 479-504.

16 Christian Joerges/Jürgen Neyer: Transforming strategic interaction into deliberative problem-solving: European comitology in the foodstuff sector, in: Journal of European Public Policy 4/1997, S. 609-625.

17 Erik Oddvar Eriksen/Jarle Weigård: Conceptualizing politics. Strategic or communicative action, in: Scandinavian Political Studies 3/1997, S. 219-241. Erik Oddvar Eriksen/John Erik Fossum: Democracy through strong publics in the European Union?, in: Journal of Common Market Studies 3/2002, S. 401-424.

18 Kerstin Jacobsson/Asa Vifell: Soft governance, employment policy and committee deliberation, in: Erik Oddvar Eriksen (Hrsg.): Making the European polity. Reflexive integration in the EU, London 2005, S. 214-236.

19 Paul Magnette/Kalypso Nicolaïdis: The European convention. Bargaining in the shadow of rhetoric, in: West European Politics 3/2004, S. 381-404.

20 Christian Joerges: Transnationale „deliberative Demokratie“ oder ,deliberativer Suprantionalismus“? Anmerkungen zur Konzeptualisierung legitimen Regierens jenseits des Nationalstaats bei Rainer Schmalz-Bruns, in: Zeitschrift für Internationale Beziehungen 1/2000, S. 145-161.

21 Vgl. hierzu Jürgen Neyer: The deliberative turn in integration theory, in: Journal of European Public Policy 5/ 2006, S. 779-791. 


\section{Supranationalität als struktureller Rahmen politischen Handelns}

Die Struktur politischen Handelns im europäischen Mehrebenensystem wird von seinem institutionellen Rahmen geprägt. Dieser setzt sich aus zwei Elementen zusammen: einer vertikalen und supranational geprägten Rechtsordnung, die normative Vorgaben für politisches Handeln macht und einer mitgliedstaatlich geprägten horizontalen Gewaltordnung, die von zentraler Bedeutung für die Umsetzung der normativen Vorgaben ist. ${ }^{22}$ Die Mitgliedstaaten der Europäischen Gemeinschaft sind keiner unabhängigen Gewalt untergeordnet und bleiben damit in eine lediglich horizontale Gewaltstruktur eingebunden. Gleichzeitig aber sind sie Mitglieder einer übergreifenden Rechtsgemeinschaft und somit in eine vertikale Rechtsstruktur integriert. Eine derartige vertikale Rechtsstruktur unterscheidet sich grundlegend von einer internationalen Rechtsstruktur. Die mitgliedstaatlichen Regierungen haben sich über die Instrumente der qualifizierten Mehrheit, des Anwendungsvorrangs europäischen vor nationalen Rechts und der Direktwirkung europäischen Rechts der Fähigkeit entledigt, die Gültigkeit außerstaatlichen Rechts auf ihrem Hoheitsgebiet kontrollieren zu können. Sie haben damit bewusst die Ausübung souveräner Rechte mit dem Vorbehalt ihrer Gemeinschaftsverträglichkeit versehen. Eine supranationale Ordnung unterscheidet sich ebenfalls grundlegend vom Nationalstaat. Die supranationalen Institutionen und ihre Rechtsnormen sind dem Staat zwar normativ übergeordnet, da sie autoritative Rechtsentstehung und -auslegung betreiben. Gleichzeitig aber verfügt nur der Nationalstaat über die Ressourcen, diese Regeln auch mit Zwang durchzusetzen.

Eine supranationale Ordnung darf trotz ihrer vertikalen Rechtsordnung und trotz der vergleichsweise geringen Kontrolle der Regierungen über den Prozess der Rechtsentstehung und -anwendung nicht mit einer staatlichen Ordnung gleichgesetzt werden. Der Europäische Gerichtshof hat in letzter Konsequenz keine Möglichkeit, seine Entscheidungen zwangsweise gegen die Mitgliedstaaten durchzusetzen, sondern muss darauf vertrauen, dass sie von den zuständigen mitgliedstaatlichen Stellen auch akzeptiert werden. Auch die Frage, ob ein nationales Gericht eine Entscheidung überhaupt dem Europäischen Gerichtshof vorlegt, steht in einer direkten Beziehung zu akzeptanzrelevanten Überlegungen: jeder Vorlageakt setzt immer voraus, dass ein Gericht subjektiv zu der Ansicht kommt, dass die strittige Frage in die Kompetenz europäischen Rechts fällt und eine entsprechende Anfrage an den Europäischen Gerichtshof stellt. Wo diese unterbleibt, da findet auch keine Anwendung europäischen Rechts statt. Hierbei handelt es sich nicht um eine bloß theoretisch relevante Problematisierung; sowohl die historische Entwicklung des Vorabentscheidungsverfahrens als auch seine praktische Verwendung zeigen, dass die mitgliedstaatliche richterliche Praxis hier durchaus Ermessensspielraum hat. ${ }^{23}$ Supranationalität ist eine Form der rechtspolitischen Integration, die wesentlich davon abhängig ist, dass sowohl ihre gouvernementalen Adressaten als auch die mitgliedstaatlichen Gerichte in einen kooperativen Prozess der Begründung und Überzeugung eingebunden werden und die daher nur in engen Grenzen hierarchische Elemente aufweisen kann.

22 Ganz ähnlich schon Joseph H.H. Weiler: The community system: The dual character of supranationalism, in: Yearbook of European Law 1/1981, S. 267-306, hier S. 267.

23 Karen J. Alter: Establishing the supremacy of European law: The making of an international rule of law in Europe, Oxford 2001. 


\section{Demokratische Regierungen sind die wichtigsten Akteure}

Regierungen müssen sich im europäischen Mehrebenensystem die politische Bühne mit anderen Akteuren teilen. Die Europäische Kommission, das Parlament und der Europäische Gerichtshof sind ebenso von zentraler Bedeutung für das Verständnis europäischer Politik wie lobbyistische Verbände und Nichtregierungsorganisationen. In einer theoretisch fokussierten und damit dem Ziel der konzeptionellen Sparsamkeit verschriebenen Betrachtung, ist es gleichwohl notwendig, unterschiedliche Akteursgruppen nach ihrer relativen Bedeutung zu unterscheiden. Relative Bedeutung erhalten in der Politik grundsätzlich solche Akteure, die über relevante Ressourcen verfügen, um die politische Agenda bestimmen und Entscheidungen beeinflussen zu können. In der Europäischen Gemeinschaft scheint hier auf den ersten Blick sowohl der Kommission als auch dem Parlament eine zentrale Bedeutung zuzukommen. Die Kommission hat das alleinige Vorschlagsrecht und das Parlament über das Mitentscheidungsverfahren ein faktisches Vetorecht für Rechtsakte. Bei einer genaueren Betrachtung weicht die These einer zentralen Machtstellung beider Institutionen allerdings sehr schnell auf. Die generellen Linien der Politik werden in der Europäischen Union vom Europäischen Rat festgelegt und der Kommission kommt in den meisten Fällen lediglich die Aufgabe zu, die generelle Agenda der Mitgliedstaaten in konkrete Rechtsakte umzusetzen. Gegen die Mitgliedstaaten kann die Kommission keine langfristige Politik machen. Auch das Recht des Parlamentes, Rechtsvorschläge des Rates zu blockieren, erscheint schon sehr viel weniger eindrucksvoll, wenn wir es in Relation dazu setzen, dass dieses Recht auf die Europäische Gemeinschaft beschränkt ist und in der Gemeinsamen Außen- und Sicherheitspolitik (GASP) sowie der Polizeilichen und Justiziellen Zusammenarbeit in Strafsachen (PJZS) nicht greift.

Die Übertragung von Kompetenzen auf die europäische Ebene bedeutet daher nicht die Aufgabe des Souveränitätsanspruches der Mitgliedstaaten, sondern lediglich ,die Bereitschaft, Souveränitätsrechte in einem Verbundsystem wahrzunehmen“. ${ }^{24}$ Auch die mitgliedstaatliche Akzeptanz von supranationalen Normen ist nur ungenügend verstanden, wenn sie als reiner Ausdruck einer gouvernemental wahrgenommenen Rechtspflicht betrachtet wird. Sie muss vielmehr als ,,autonomer freiwilliger Akt" verstanden werden, der die grundsätzlich jederzeit widerrufbare Einsicht in die Sinnhaftigkeit der europäischen Rechtsordnung zum Ausdruck bringt. ${ }^{25}$ Dieser im politischen Alltagsgeschäft von der Routine der Rechtsbefolgung überdeckte Tatbestand kommt deutlich darin zum Ausdruck, dass supranationales Recht eben nicht zwangsbewehrt ist und es jedem Mitgliedstaat in letzter Konsequenz faktisch freisteht, den innerstaatlichen Rechtscharakter europäischen Rechts zu widerrufen und damit die europäische Rechtsgemeinschaft außer Kraft zu setzen. ${ }^{26}$

Trotz ihrer zentralen Rolle im europäischen politischen Prozess dürfen die Regierungen der Mitgliedstaaten nicht als autonome Akteure missverstanden werden. Als Repräsentanten demokratischer Gesellschaften müssen sie sich öffentlich für die von ihnen verfolgten Politiken rechtfertigen. Das schließt ebenfalls eine Beschränkung auf die bloße Befriedigung der politischen Wünsche dominanter Interessengruppen aus. Demokratische Regierungen sind

24 Rainer M. Lepsius: Die Europäische Union als Herrschaftsverband eigener Prägung, Harvard Jean Monnet Working Paper 7/2000, Harvard Law School, Cambridge 2000, S. 7.

25 Joseph H.H. Weiler: Federalism and constitutionalism: Europe's Sonderweg, Harvard Jean Monnet Working Paper 10/2000, Harvard Law School, Cambridge 2000, S. 13.

26 Es gibt in der Rechtswissenschaft allerdings ebenfalls die Ansicht, dass die Mitgliedstaaten über diese Kompetenz nicht mehr verfügen, da die EU eine Union von Staaten und Bürgern sei und die Mitgliedstaaten daher nicht eigenständig Rechte der Unionsbürgern liquidieren dürften. Ich verdanke diesen Hinweis einem der Gutachter. 
der permanenten öffentlichen Kritik ausgesetzt und können nur sehr beschränkt parteiliche Politik machen. Um wieder gewählt zu werden müssen sich Regierungen, auch wenn sie aus kleineren Parteien zusammengesetzt sind, faktisch als Volksparteien verhalten. Das Gesellschaftsmodell der deliberativen Theorie ist damit die kritische und Rechtfertigung einfordernde Öffentlichkeit.

In der Realität werden hier oft Abstriche gemacht werden müssen. Es ist empirisch sicherlich zutreffend, dass die von Politikern wahrgenommene öffentliche Meinung in vielen Fällen identisch mit der veröffentlichten Meinung ist. Die veröffentlichte Meinung ist zudem Gegenstand von Skandalisierungen und ökonomischen Interessen und weicht mitunter gravierend von der idealtypisch gedachten Agora ab. Das Modell einer Rechtfertigung einfordernden Öffentlichkeit wird hierdurch allerdings nicht grundsätzlich in Frage gestellt. Regierungen würden durch diese Einschränkungen nur dann weniger stark rechenschaftspflichtig werden, wenn es ein hohes $\mathrm{Maß}$ an Abhängigkeit der veröffentlichten Meinung von Regierungsinteressen oder gar eine enge personelle Verflechtung zwischen beiden gäbe. Dieses ist zwar nicht grundsätzlich ausgeschlossen, lässt sich heute allerdings nur in wenigen Mitgliedstaaten beobachten.

\section{Diskurs als Prozess}

Das wesentliche Bindungsglied zwischen den europäischen Regierungen sowie zwischen den Regierungen und den von ihnen repräsentierten Gesellschaften ist daher der permanente politische Diskurs und die konstruktive Auseinandersetzung. Europa verstehen kann daher auch nicht bloß bedeuten, Machtasymmetrien und Hierarchien zu analysieren oder technische Antworten auf ebensolche Probleme zu beschreiben. Europa wird im Kern von verständigungsorientierten politischen Diskursen zusammengehalten und hört dort auf zu funktionieren, wo die Grenze der Verständigung zwischen Gesellschaften und den sie repräsentierenden Regierungen erreicht ist.

Die deliberative Integrationstheorie setzt genau hier an und unterscheidet politische Diskurse danach, welches Potenzial der Verständigung sie haben. Zwei Grundtypen sind die Verhandlung und die Argumentation. ${ }^{27}$ Die Verhandlung lässt sich als das Austauschen von Versprechen und Drohungen verstehen und führt insbesondere in bilateralen Konstellationen oftmals zügig zu Einigungen. Der Verhandlungsmodus weist allerdings in multilateralen Konstellationen auch ein gravierendes Problem auf. Wenn die Kosten der Überwachung mitgliedstaatlicher Rechtseinhaltung hoch und die Fähigkeiten zur Erzwingung von Rechtsgehorsam niedrig sind, müssen Regelungsadressaten eine eigene Motivation zur Rechtsbefolgung haben. Diese Motivation setzt im Normalfall voraus, dass eine Regelung von ihren Adressaten für inhaltlich angemessen gehalten wird und dass alle relevanten Argumente im Rechtssetzungsprozess abgewogen und entsprechend geprüft wurden. In internationalen Organisationen wird daher zumeist nicht nur verhandelt, sondern ebenfalls argumentiert. Der von Wallace und Hayes-Renshaw beschriebene ,instinktive Rekurs auf den Konsens “28 und die Sparsamkeit, mit der Abstimmungen im Rat eingesetzt werden, finden hier ihren tieferen Grund. Europa ist nur dann effektiv, wenn es überzeugt.

27 Ausführlich hierzu Thomas Saretzki: Wie unterscheiden sich Argumentieren und Verhandeln? Definitionsprobleme, funktionale Bezüge und strukturelle Differenzen von zwei verschiedenen Kommunikationsmodi, in: Volker von Prittwitz (Hrsg.): Verhandeln und Argumentieren. Dialog, Interesse und Macht in der Umweltpolitik, Opladen 1996, S. 19-39.

28 Hayes-Renshaw/Wallace: Executive power in the European Union, 1995. 
Überzeugen ist allerdings gerade in einem multikulturellen Kontext mit unterschiedlichen Regelungstraditionen und ökonomischen Anpassungskosten außerordentlich schwierig. Delegierte werden nicht nach Brüssel geschickt, um sich überzeugen zu lassen, sondern sollen nationale Positionen mit allem angemessenen Nachdruck vertreten. Delegierte, die ihren Ministern zu Hause berichten, dass sie sich von den besseren Argumenten anderer Delegationen haben überzeugen lassen, werden selten freudig begrüßt. Das europäische Recht hat hier eine außerordentlich wichtige Funktion als Anreizstruktur für die Transformation von Verhandlungen in Argumentationen und damit die Ermöglichung von Überzeugungsprozessen. Der zentrale Anreiz für diesen transformativen Akt wird vom europäischen Recht über die Konditionalisierung der Legitimität von gouvernementalen Präferenzen erzeugt: Gouvernementale Präferenzen werden in einer rechtsbasierten Gemeinschaft wie der Europäischen Gemeinschaft nicht mehr als intrinsisch legitimer Ausdruck innerstaatlicher Meinungsbildungsprozesse verstanden, sondern nur noch dann und nur insoweit als legitim betrachtet, wie sie in widerspruchsfreier Übereinstimmung mit dem Vertragsrecht stehen. Regierungen werden dadurch im politischen Diskurs genötigt, Gründe für ihre Präferenzen anzugeben und deren widerspruchsfreie Übereinstimmung darzulegen.

Die Konditionalisierung der Legitimität gouvernementaler Präferenzen führt im Ergebnis dazu, dass dort argumentatives Handeln eingefordert wird, wo ansonsten die Machtdifferenzen der Staaten und der Verhandlungsprozess die Politik bestimmen würden. Durch das Recht werden zwar sicherlich nicht alle Machtasymmetrien herausgefiltert. Und ebenfalls wird es immer wieder vorkommen, dass ausschließlich national motivierte Anliegen vorgetragen werden. Gleichzeitig aber führt das Initiativmonopol der Kommission dazu, dass derartige Anliegen kaum Aussichten haben, zur Grundlage für einen Rechtsakt zu werden. Auch in den anwendungsbezogenen Bereichen, wie der Notifikation von handelsrelevanten nationalen Rechtsakten, haben argumentativ nicht rechtfertigbare Handlungen kaum eine Chance, der Kontrolle durch die Kommission und die anderen Mitgliedstaaten zu entgehen, sondern würden im Zweifelsfall Gegenstand einer rechtlichen Prüfung durch den Europäischen Gerichtshof werden. Empirisch lässt sich diese Konditionalisierung der Legitimität von Präferenzen in einer Vielzahl von Bereichen beobachten. Das wohl bekannteste Beispiel hierfür ist die seit dem Urteil ,Cassis de Dijon ${ }^{29}$ geltende Grundregel der Rechtfertigungsbedürftigkeit nationaler Beschränkungen des freien Handels. Artikel 28 EGV sieht hierzu vor, dass alle mengenmäßigen Beschränkungen sowie alle Maßnahmen gleicher Wirkung grundsätzlich verboten sind wenn sie sich nicht auf die Ausnahmetatbestände des Artikels 30 EGV berufen können. Hierzu gehören unter anderem Einfuhrbeschränkungen, die aus Gründen der öffentlichen Sicherheit, des Verbraucherschutzes oder des Umweltschutzes verhängt wurden. Handelsbeschränkende nationale Maßnahmen, die sich nicht unter diese Gründe fassen lassen, sind untersagt.

Während Machtasymmetrien und nationale Egoismen in einer intergouvernementalen Struktur dominante Phänomene sind, haben sie in einer supranationalen und damit rechtsbasierten Struktur nur noch beschränkte Auswirkungen auf den Gehalt legislativer Prozesse. In dem argumentationsbasierten Repräsentationsmodus supranationaler Politik ist es von untergeordneter Bedeutung, ob ein Argument von einem großen oder kleinen Staat vorgebracht wurde. Wichtig ist ausschließlich die Frage, ob das vorgebrachte Argument rechtlich belastbar ist. Was letztlich zählt sind nicht akteursbezogene Merkmale und die Glaubhaftigkeit von Drohungen und Versprechen, sondern die Güte eines Arguments. Der territoriale Repräsentationsmodus der intergouvernementalen Politik wird in einer supranationalen Struktur

29 Europäischer Gerichtshof, Urteil vom 20.2.1979, Rs. 120/78, Slg. 1979, 649. 
somit von einem argumentationsbasierten Repräsentationsmodus gleichzeitig eingerahmt und diszipliniert. Durch die Konditionalisierung der Legitimität gouvernementaler Präferenzen und ihre Einbindung in die Pflicht zur Rechtfertigung beuten supranationale Institutionen das Verständigungspotenzial diskursiver Verfahren aus und verleihen sachhaltigen Anliegen auch dann Nachdruck, wenn sie im Widerspruch zu machtvollen Interessen stehen. Empirisch ist hier natürlich auch wieder eine Relativierung einzufügen. Machtbasierte Verhandlungsprozesse werden niemals vollständig vom Recht in verständigungsorientierte Argumentationen überführt. Das Recht mildert die Asymmetrien ab, aber es löst sie nicht auf.

Es gibt in der Europäischen Gemeinschaft noch einen zweiten wirkungsvollen Mechanismus, der diskursive Verfahren befördert. Das in den meisten Fällen zur Anwendung kommende qualifizierte Mehrheitsverfahren schafft für Regierungen immer dann einen Anreiz zum vernünftigen Argumentieren, wenn sie bisher unentschiedene Staaten von der Richtigkeit der eigenen Position überzeugen wollen. Sowohl die Befürworter als auch die Gegner einer Regelung müssen in einem qualifizierten Mehrheitsverfahren den Versuch unternehmen, eine breite Koalition für die Unterstützung eines Projektes zu schmieden oder aber eine Gruppe von Staaten zusammenzubringen, die sich diesem Projekt entgegenstellt. Für das Schmieden von Koalitionen lassen sich grundsätzlich natürlich nicht nur Argumente, sondern auch Drohungen und Versprechungen einsetzen. Qualifizierte Mehrheitsverfahren gehen daher nicht notwendigerweise mit guten Argumenten und rechtfertigungsorientierten Diskursen einher. Gleichzeitig aber sind Versprechungen und Drohungen nicht für alle Staaten gleichermaßen glaubwürdig formulierbar. Gerade kleineren Staaten fehlen oftmals die notwendigen politischen und ökonomischen Ressourcen. Hinzu kommt, dass auch für die größeren Staaten die Kosten verhandlungsbasierter Strategien proportional zu der Anzahl von Staaten zunehmen, die zu einer Koalition zusammengeführt werden sollen: Um zwei Staaten auf dem Verhandlungsweg zu überzeugen, muss ein aktiv verhandelnder Staat beiden Parteien entweder kostenintensive Versprechungen machen oder entsprechend glaubwürdige Drohungen artikulieren. Bei vier Staaten verdoppeln sich die Kosten, da nunmehr für eine doppelt so große Anzahl von Akteuren positive oder negative Anreize geschaffen werden müssen. Wenn wir diesen Gedanken konsequent fortsetzen, kommen wir schnell zu der Einsicht, dass Verhandlungsstrategien insbesondere in bilateralen Situationen attraktive Instrumente zur Realisierung von politischen Koalitionen sind, während sich diese Attraktivität mit zunehmender Anzahl von Staaten, die ,überzeugt“ werden sollen, schnell verringert. Im Gegenzug gewinnen argumentationsbasierte Strategien, die an gute Gründe appellieren, an Attraktivität, da die Kosten eines Arguments unabhängig von der Anzahl seiner Adressaten sind. Qualifizierte Mehrheitsverfahren und die Notwendigkeit der Realisierung staatenübergreifender politischer Koalitionen haben damit gerade bei einer größeren Anzahl von Staaten eine hohe Wahrscheinlichkeit, zu argumentativen Interaktionsformen zu führen.

\section{Die deliberative Erklärung der Krise der Europäischen Union}

Deliberative Verfahren sind in der Europäischen Union nicht überall gleichermaßen ausgeprägt. Grundsätzlich lassen sie sich eher in der Europäischen Gemeinschaft als in intergouvernementalen Bereichen der Europäischen Union beobachten, da nur hier eine entsprechende Dichte von justiziablen Rechtsnormen existiert. Aber auch in der Europäischen Gemeinschaft sind deliberative Verfahren stark asymmetrisch ausgeprägt. Während in den intergouvernementalen Beziehungen die rechtlichen Vorgaben für die Rechtfertigung von Politik fest institutionalisiert und über die Zeit eher dichter geworden sind, lässt sich in der Anbindung von Regierungshandeln an die Kontrolle durch nationale Öffentlichkeiten und 
Parlamente ein gegenteiliger Prozess beobachten. Die Verlagerung von politischen Kompetenzen auf die europäische Ebene läuft der Etablierung von institutionellen Strukturen, die innerstaatliche Rechtfertigung und Begründung einfordern, weit davon.

Das Auseinanderfallen von politischen Kompetenzen und institutionell abgesicherten Rechtfertigungspflichten der Regierungen gegenüber ihren Gesellschaften ist besonders weitreichend in der intergouvernementalen GASP und der PJZS. Hier werden grundlegende Fragen der inneren und äußeren Sicherheit behandelt, ohne dass das Europäische Parlament ein Mitbestimmungsrecht hat oder der Europäische Gerichtshof für die rechtliche Kontrolle zuständig ist. Dort, wo Verhandlungen außerhalb öffentlicher Kontrolle und rechtlicher Überprüfung stattfinden, sind weder effektive Argumentationen noch das Einlösen von politischen Rechtfertigungspflichten zu erwarten. Ohne die öffentliche Rechtfertigung von Politik aber entsteht leicht Misstrauen gegenüber der Macht. Misstrauen aber führt in einer Demokratie wiederum gewöhnlich zu politischem Widerstand. Legitimitätskrisen sind damit letztlich Vertrauenskrisen, die sich aus der zunehmenden Unsicherheit der Gesellschaften gegenüber der Art der Wahrnehmung politischer Kompetenzen speisen.

Für die Erklärung der aktuellen Krise der Union ist dieser Zugriff ganz offensichtlich relevant. Nach wie vor ist die Europäische Union in weiten Teilen ein technokratisch-intergouvernementales Regime, in dem die wichtigsten Entscheidungen von den Regierungen hinter verschlossenen Türen und unter Ausschluss breiterer gesellschaftlicher Kreise getroffen werden. ${ }^{30}$ Es ist zwar zutreffend, dass der Ministerrat gelegentlich öffentlich tagt und ebenfalls ist es richtig, dass die Kompetenzen des Parlamentes über die Zeit immer weiter ausgebaut wurden. Gleichzeitig aber wurde der Europäische Rat in seiner Rolle als Agenda setzende Institution bestärkt und wurde dem Parlament die Kompetenz verweigert, eigenständig die politische Agenda der Europäischen Union bestimmen zu können. Das Parlament ist daher letztlich lediglich in seiner Funktion gestärkt worden, den Mitgliedstaaten dabei zu helfen, ihre im Europäischen Rat gefundene Agenda umzusetzen. Die wichtigsten politischen Entscheidungen und Weichenstellungen der europäischen Politik finden aber nicht im Parlament - oder auch nur zwischen Parlament und Ministerrat -, sondern hinter den verschlossenen Türen des Europäischen Rates statt. Im Europäischen Rat werden die Grundzüge der europäischen Außenpolitik formuliert, werden die wichtigsten Beamten der europäischen Institutionen ernannt, werden vertragsverändernde Regierungskonferenzen initiiert und verabschiedet, und wird de facto über die Aufnahme neuer Mitgliedstaaten entschieden.

Es ist daher auch nicht überraschend, dass fast alle der wirklich wichtigen politischen Weichenstellungen der letzten fünfzehn Jahre auf rein intergouvernementale Beschlüsse zurückgehen: Die mitgliedstaatlichen Exekutiven handelten die Wirtschafts- und Währungsunion, den Stabilitätspakt und die Osterweiterung der Europäischen Union unter sich aus. Der Umbau der Union in einen Raum der Freiheit, der Sicherheit und des Rechts und die hiermit einhergehende Ausdehnung gouvernementaler Kompetenzen in den Bereich der inneren Sicherheit wurde den europäischen Gesellschaften vom Europäischen Rat als eine notwendige Reaktion auf die Terrorismusgefahr präsentiert und ebenso per intergouvernementaler Deklaration verkündet wie der Ausbau der militärischen Komponente der GASP und die hiermit einhergehende schleichende Militarisierung der europäischen Außenpolitik. In den wichtigen Fragen der europäischen Politik gibt es daher weder eine institutionalisierte

30 Jürgen Neyer/Michael Schroeter: Deliberative Europe and the rejected constitution, in: Erik Oddvar Eriksen/ Christian Joerges/Florian Rödl (Hrsg.): Law and democracy in the post-national-union, Arena-Report 1/2006, S. 61-90. 
Opposition noch publikumswirksame Foren der Auseinandersetzung mit abweichenden Meinungen.

Auch die Reaktion der Europäischen Union auf die Herausforderung der Globalisierung ist nicht gerade von öffentlicher Teilhabe geprägt. Die Politiken der Union beschränken sich weitestgehend auf Maßnahmen zur Förderung von Wettbewerbsfähigkeit und neuen Technologien und haben keinerlei Sensibilität für die sozialen Ängste ihrer Bürger. Die Entwicklung einer europäischen Beschäftigungsstrategie ist über unverbindliche Empfehlungen nicht heraus gekommen. Aufgrund der einseitigen Konzentration der europäischen Politik auf die Beförderung der negativen Integration gilt der Begriff der Europäisierung für viele als synonym für die sozialen Verwerfungen der Globalisierung. Die Osterweiterung steht im Bewusstsein vieler Menschen nicht für die historische Überwindung der Teilung des Kontinentes, sondern für erhöhten Wettbewerbsdruck und weiteren Arbeitsplatzverlust. Aus der ehemaligen Hoffnung auf eine Antwort auf die Globalisierung ist das trojanische Pferd der Globalisierung geworden.

Es geht hier nicht darum, einzelne dieser Politiken inhaltlich zu kritisieren. Für alle lassen sich vielmehr durchaus gute inhaltliche Gründe vorbringen. Das zentrale Problem liegt tiefer. Mit der Osterweiterung, dem Stabilitätspakt, der Militarisierung der GASP und der Globalisierung hat die Union die ,Nichtsichtbarkeitsschwelle "überschritten und den ehemals so wirkungsvollen permissiven Konsens in Frage gestellt. Die Europäische Union ist relevant und politisch erwachsen geworden. Damit aber hat sie sich automatisch auch mit wachsender Kritik auseinanderzusetzen und muss auf sie eingehen.

Hier kann die Europäische Union ausnahmsweise mal von den Mitgliedstaaten lernen: Die mitgliedstaatlichen Demokratien haben sich in der Vergangenheit gerade deswegen als eine außerordentlich stabile und legitime Form der Gestaltung politischer Ordnung erwiesen, weil sie der kritischen Opposition gegen spezifische Politiken einen prominenten und öffentlichkeitswirksamen Platz im politischen System eingeräumt haben. Die Permanenz von gleichzeitig kritischer und konstruktiv eingebundener Opposition ist eines der wichtigsten Erfolgsrezepte der Demokratie. Die alltägliche Kritik und Rechtfertigung im Parlament, in den Medien und über Regierungserklärungen hat die außerordentlich wichtige Funktion, dass sich die Opposition an einzelnen Politiken abarbeiten kann und sich damit nicht gegen das System als solches wenden muss. In der Europäischen Union aber fehlt genau diese Alltäglichkeit der institutionalisierten Auseinandersetzung mit Kritik.

Es wird Zeit, dass sich die europäischen Entscheidungsverfahren dem anpassen. Der wichtigste Schritt auf diesem Weg ist es, der Opposition gegen spezifische europäische Politiken einen institutionellen Platz einzuräumen, damit die Opposition gegen das europäische Integrationsprojekt ein Randphänomen bleibt. Wer die Deliberation und damit auch die kritische Opposition in Europa verhindert, droht hingegen das europäische Projekt selbst zu gefährden. Es kann daher auch überhaupt nicht überzeugen, wenn mitgliedstaatliche Regierungen heute versuchen, den Reformvertrag als technisches Instrument klein zu reden und ohne die Durchführung von Referenden von ihren nationalen Parlamenten annehmen zu lassen. Mit den Referenden zum Verfassungsvertrag hat sich in Europa die Norm etabliert, dass größere Integrationsfortschritte der explizit erteilten gesellschaftlichen Zustimmung bedürfen. Mit der inzwischen eingeübten Praxis der Durchführung von Referenden ist den Mitgliedstaaten die Flasche mit dem eingeschlossenen Geist der Bürgerteilhabe aus der Hand gefallen. Sie haben damit eine normative Realität geschaffen, hinter die sie nur noch um den Preis einer weiteren Entfremdung der Europäischen Union von ihren Bürgern werden zurückfallen können. Wenn dieses vermieden werden soll - und das muss es, wenn die Europäische Union längerfristig als legitim anerkannt bleiben will - dann werden die mitglied- 
staatlichen Regierungen nicht umhin kommen, ihr Umgehen mit der Europäischen Union grundlegend zu ändern. Eine zentrale politische Forderung muss daher heute lauten, mit dem Europäischen Rat das eigentliche Machtzentrum der Europäischen Union zu parlamentarisieren und diesen reformierten Europäischen Rat die Kommission wählen zu lassen. Nur so lässt sich der notwendige konstruktive Streit in die Union importieren und damit gewährleisten, dass er sich nicht als unkonstruktive Frustration gegen das System als solches wendet.

\section{Fazit}

Die deliberative Integrationstheorie ist eine Antwort auf die Defizite der aktuellen Integrationsforschung. Im Gegensatz zum Intergouvernementalismus betont die deliberative Theorie die zentrale Rolle der mitgliedstaatlichen Gesellschaften im Integrationsprozess sowie des europäischen Rechts als Verständigung förderndes Medium. Die deliberative Integrationstheorie setzt sich ebenfalls vom Funktionalismus ab. Sie sieht keinen Automatismus der Integration vor, sondern rechtsbasierte Entscheidungsprozesse, die sowohl zu einem Mehr als auch einem Weniger an Integration führen können. Integrationsfortschritte werden in einer deliberativen Perspektive als rechtfertigungsbedürftige Verlagerungsprozesse von politischer Macht verstanden. Mehr Integration verlangt immer auch ein Mehr an Rechtfertigung. Dort wo diese Rechtfertigung unterbleibt oder als unglaubwürdig wahrgenommen wird, entsteht Misstrauen und letztlich politischer Widerstand.

Die wesentliche Stärke einer deliberativen Integrationstheorie liegt in ihrer gesellschaftstheoretischen Aktualität. Sie basiert auf einem Gesellschaftsverständnis, das die Begriffe der Öffentlichkeit und der Demokratie nicht systematisch ausblendet, sondern ihnen einen zentralen analytischen Platz einräumt. Damit wird sie zu einem Instrument, das sowohl die Realität zu erklären beanspruchen als auch normative Reflexionen anleiten kann. Die Grenzen der Erklärungskraft einer deliberativen Integrationstheorie liegen dort, wo ihre Grundannahmen empirisch unzutreffend sind. Wo die Gesellschaften der europäischen Mitgliedstaaten die europäische Politik nicht kritisch beobachten und wo von Mandatsträgern keine Rechtfertigung für politisches Handeln eingefordert wird, da ist auch nicht anzunehmen, dass deliberative Strukturen entstehen. Die Erklärungskraft einer deliberativen Theorie hängt damit letztlich von dem Ausmaß ab, zu dem Öffentlichkeit entweder bereits besteht oder zumindest Rechtfertigung eingefordert wird. Wo aber weder das eine noch das andere existiert, da sind auch die Grenzen der Erklärungskraft einer deliberativen Integrationstheorie erreicht. 